\title{
Evaluation of Corneal Oedema - Tools we Have and Those Under Investigation
}

\author{
Yu-Chi Liu, ${ }^{1,2,3}$ Lin Ke, ${ }^{4}$ Jodhbir S Mehta ${ }^{1,2,3}$ \\ 1. Tissue Engineering and Stem Cell Group, Singapore Eye Research Institute, Singapore; 2. Department of Cornea and External Eye Disease, \\ Singapore National Eye Centre, Singapore; 3. Ophthalmology and Visual Sciences Academic Clinical Program, Duke-NUS Medical School, \\ Singapore; 4. Institute of Materials Research and Engineering, Agency for Science, Technology and Research, Singapore
}

DOI: https://doi.org/10.17925/EOR.2019.13.2.76

\begin{abstract}
$\mathrm{C}$ orneal oedema is a common sign of acute or chronic corneal disease resulting from various aetiologies such as corneal endothelial dystrophy, prior surgery such as cataract surgery, metabolic disorders, toxicity or hypoxia. Measurement of central corneal thickness (CCT) is an objective and surrogate assessment for the evaluation of corneal oedema. The commonly used tools for the CCT measurements include ultrasonic pachymetry, anterior segment optical coherence tomography, specular microscopy and corneal tomography. Terahertz and mid-infrared technology have emerged recently for their applications on corneas. Instead of measuring CCT, they detect the changes of corneal water concentrations because their light wave spectrum is sensitive to water. The utility of these imaging systems as an adjuvant tool for the evaluation of corneal oedema, will be discussed in this article.
\end{abstract}

\section{Keywords}

Corneal edema, corneal disease, corneal endothelial dystrophy, central corneal thickness, CCT, Terahertz, mid-infrared technology

Disclosure: Yu-Chi Liu, Lin Ke and Jodhbir S Mehta have nothing to disclose in relation to this article. Review Process: Double-blind peer review

Authorship: All named authors meet the International Committee of Medical Journal Editors (ICMJE) criteria for authorship of this manuscript, take responsibility for the integrity of the work as a whole, and have for the integrity of the work as a whole, and have
given final approval to the version to be published. Compliance with Ethics: This study involves a review of the literature and did not involve any studies with human or animal subjects performed by any of the authors.

Received: 25 October 2019

Accepted: 9 January 2020

Citation: European Ophthalmic Review, 2019;13(2):76-80

Corresponding Author: Yu-Chi Liu, 20

College Road, Discovery Tower, Level 12,

Singapore 169856. E: liuchiy@gmail.com

Support: No funding was received in

the publication of this article.
The cornea is a transparent and avascular tissue. The optical transparency, which is directly related to the state of corneal hydration, is essential for high resolution of vision. Corneal hydration is controlled by (1) factors that draw water into the cornea, including the intraocular pressure and swelling pressure from stromal glycosaminoglycans; ${ }^{1,2}$ and (2) factors that prevent the flow of water into the cornea, i.e., the intact corneal epithelial and endothelial barriers. The functioning corneal metabolic endothelial pump, which is linked to an ion-transport system, is controlled by temperature-dependent enzymes such as $\mathrm{Na}^{+}, \mathrm{K}^{+}$-APTase. ${ }^{1}$ Corneal hydration varies from the anterior to posterior of corneas, with increasing saturation to the endothelium. When the endothelial cells are impaired and the number of damaged endothelia exceeds the threshold necessary to maintain the functioning of the cornea, fluid leaks into the corneal stroma, resulting in corneal oedema. Folds in Descemet's membrane are usually first seen when corneal thickness increases by $10 \%$ or more. ${ }^{1}$ As the corneal oedema progresses, the oedema extends from the stromal space to epithelial layer, leading to epithelial oedema and bullae. When epithelial oedema occurs, the corneal thickness usually has exceeded $700 \mu \mathrm{m} .{ }^{1}$

Corneal oedema results from various aetiologies (Table 1), including endothelial dystrophy; previous surgery, such as cataract surgery or glaucoma filtrating surgery; mechanical trauma; metabolic disorders; toxicity or hypoxia. ${ }^{1,3,4}$

\section{Evaluation of corneal oedema}

Clinically, the diagnostic workup for corneal oedema starts with slit lamp examination, in which an oblique and thin light beam on oblique viewing of the optical section of the corneas can be used to estimate the corneal thickness. Measurement of central corneal thickness (CCT) is an indirect and surrogate measure for the evaluation of corneal hydration status. Ultrasound pachymetry has been used for more than 30 years and is one of the most commonly used methods for measuring $\mathrm{CCT}$, by estimating the time difference between echoes of ultrasound waves reflected from the anterior and posterior surface of the cornea. ${ }^{5}$ However, its reliability can be limited due to the susceptibility to confounding factors such as topical anaesthesia, and requiring individual user skill to place the probe as perpendicularly as possible on the central corneal apex. Moreover, it requires cornea-probe contact. ${ }^{6}$ The use of topical anaesthesia has been reported to cause cornea thickening up to $31 \mu \mathrm{m} .{ }^{.}$These limitations have led to the introduction of several optical technologies that offer the advantages of a non-contact technique and objective determination of the centre of the cornea. A non-invasive and non-contact tool is especially preferred in patients who have undergone refractive surgery or corneal transplantation to avoid complications that are related to the measuring, such as corneal epithelial abrasions, laser in situ keratomileusis (LASIK) flap displacement, or potential exposure of corneal infection due to the probe contact.

Very high-frequency (VHF) digital ultrasound was introduced in 1993 and has gradually improved both in precision and in area of acquisition. It produces three-dimensional thickness maps of the 
Table 1: Aetiology of corneal oedema

\begin{tabular}{|l|}
\hline Early age onset \\
\hline Congenital glaucoma \\
\hline $\begin{array}{l}\text { Dystrophy: congenital hereditary endothelial dystrophy, posterior } \\
\text { polymorphous corneal dystrophy }\end{array}$ \\
\hline Intraocular inflammation \\
\hline Birth trauma \\
\hline Late age onset \\
\hline Acute angle-closure glaucoma \\
\hline Dystrophy: Fuchs' dystrophy, posterior polymorphous corneal dystrophy \\
\hline Hypotony \\
\hline Hypoxia, such as prolonged use of contact lens \\
\hline Intraocular inflammation \\
\hline Iridocorneal endothelial syndrome \\
\hline Infectious keratitis \\
\hline Hydrops of keratoconus \\
\hline Surgical trauma \\
\hline Toxic, such as silicone oil, chemotherapy, amantadine, chlorhexidine \\
\hline Metabolic disorders, such as myxedema, hypercholesterolemia \\
\hline
\end{tabular}

cornea over a larger, 10-mm diameter central zone. The improved signalto-noise ratio when compared to conventional ultrasound biomicroscopy allows visualisation of internal corneal lamellar interfaces. Repeatability of corneal thickness measurements using VHF digital ultrasound has been reported to be $1.68 \mu \mathrm{m} .{ }^{8,9}$

Specular microscopy is a non-contact method that assesses not only corneal endothelium morphology but also CCT. The CCT is derived based on the reflection of light waves from the corneal anterior and posterior surfaces. Computerised corneal topographers such as a Placido diskbased topographers, reflect images of multiple concentric circles that can be digitally captured and analysis performed by computer software. Although modern topography devices are able to map a large part of the anterior segment, a complete pachymetric evaluation is not possible without information on the posterior corneal surface. ${ }^{1}$ Contrary to topography, corneal tomography evaluates the whole cornea by obtaining information from both the anterior and posterior corneal surfaces. Slit-scanning elevation topography like Orbscan IIz (Bausch \& Lomb, Rochester, NY, USA) is a projective technique that measures the triangulation between the reference slit beam surface and the reflected beam captured by a camera. It combines a three-dimensional scanning slit beam system with an added advanced Placido disk attachment. Slits are projected sequentially on the cornea during image acquisition to create an overlapping pattern of scanning slits, and the final image is represented as a three-dimensional topographic map including a pachymetry map of the entire corneal surface..$^{10}$ The Pentacam ${ }^{\circledast}$ (Oculus Optikgerate $\mathrm{GmbH}$, Wetzlar, Germany) is an imaging system based on a capture method called Scheimpflug imagery, in which a single rotating Scheimpflug camera measures 25,000 elevation points and gives a three-dimensional scanning representation of the shape of the corneal surface including the measurement of corneal thickness. ${ }^{11}$ There are another three Scheimpflug-based devices, such as Galilei (Ziemer, Port, Switzerland), are available. ${ }^{10}$ Besides corneal topography and tomography, anterior segment optical coherence tomography (ASOCT) is based on low-coherence interferometry and is also non-contact. The ASOCT generates two- or three-dimensional tomographic images by measuring the echo time delay of light backscattered from tissue structures. ${ }^{12}$ The speed and scanning resolution have improved over the past decades. In time-domain optical coherence tomography (OCT), the system acquires approximately $400 \mathrm{~A}$-scans per second using six radial slices oriented 30 degrees apart, while in spectral-domain OCT, the scanning rate is approximately 20,000-40,000 scans per second, minimising unwanted motion artefact and enhancing the resolution..$^{12}$ The RTVue ${ }^{\oplus}$ (Optovue, Inc., Fremont, CA, USA), for example, provides a rapid imaging that overcomes eye movement velocity and ensures $5 \mu \mathrm{m}$ resolution and high-magnification imaging of the cornea within 0.04 seconds. ${ }^{6}$ The high measurement velocity is achieved with a stationary reference mirror, and it collects signals from the entire range of interest and analyses data using the spectral interferogram and rapid Fourier transformation. ${ }^{13}$ Besides spectral-domain OCT, swept-source OCT is also categorised as Fourier-domain OCT. Its light source is rapidly swept within the laser, allowing for the use of a point detector and high acquisition rates up to several $\mathrm{MHz}{ }^{12}$

In vivo confocal microscopes can be used to measure corneal thickness with its function 'confocal microscopy through focusing on the tandem scanning confocal microscopes'. Different layers of the cornea reflect light at different intensities, and the intensity profile Z-curve can be analysed by specialised software to measure the distance between any two points on the curve. ${ }^{14}$

A vast number of studies have been conducted to investigate the reproducibility, repeatability and interchangeability of these modalities, and several considerations have to be taken into account before making comparisons. It has been reported that specular microscopy had lower CCT readings than those obtained by ultrasound pachymetry, in normal subjects and post-LASIK patients, with the range of difference of 14.4$31.6 \mu \mathrm{m} .{ }^{15-17}$ There is also a tendency of Scheimpflug tomographers to underestimate the measurement of CCT, compared to ultrasound pachymetry, in normal corneas and patients with keratoconus, with a mean difference of 5-9 $\mu \mathrm{m}$. ${ }^{18-21}$ In general, optical readings tended to be slightly thinner than ultrasound pachymetry readings. ${ }^{17}$ Optical pachymeters, like specular microscopy or Scheimpflug scanning, require clear reflection of the anterior and posterior corneal surfaces to generate a reliable thickness measurement. Histopathologic features, such as the stromal haze or interface debris, the altered organisation of collagen fibres in keratoconus, or the irregularity of endothelium in patients with corneal oedema, affect the corneal optical properties and interrupt the pathway of light rays, while the velocity of ultrasound is less disturbed by media clarity. ${ }^{22}$ However, the contact technique of ultrasound pachymetry is prone to be confounded by the changes of corneal rigidity, such as corneal oedema or post refractive surgery. The speed of the ultrasound and the point of sound reflection may be changed in these scenarios, which in turn results in the inconsistency of the measurements. ${ }^{23.24}$ Moreover, in ultrasound pachymetry, the definite posterior corneal reflection point is not well understood:25 it can be located in front of corneal Descemet's membrane, or in front of the anterior chamber, and this may also affect the measurements.

When comparing ASOCT with Pentacam or ultrasound pachymetry, Nam et al. showed that the RTvue had better reliability and accuracy. ${ }^{6}$ There are two theoretical reasons that can explain the high reliability of corneal thickness measurement with RTVue. First, RTvue measures the central zone average of the corneal thickness instead of single-point thickness, and the central zone average is more reliable than an individual value. Second, the fast scan speed of RTVue may offer the advantages to minimise the artefact due to eye movements. 6 In ultrasound pachymetry measurements, the accuracy is examiner dependent, while in ASOCT 
Table 2: Tools evaluating cornea oedema

\begin{tabular}{|c|c|}
\hline Device & Principles \\
\hline \multicolumn{2}{|l|}{ Measure corneal thickness } \\
\hline Slit lamp biomicroscopy & - Uses an oblique and thin light beam on oblique viewing of the optical section of the corneas to estimate the corneal thickness \\
\hline Ultrasound pachymetry & $\begin{array}{l}\text { - Estimation of corneal thickness is based on the speed of sound in the normal cornea }(1,640 \mathrm{~m} / \mathrm{sec}) \text { and the time difference between } \\
\text { the ultrasound waves reflected from the anterior and posterior surface of the cornea }\end{array}$ \\
\hline Specular microscopy & - Corneal thickness is derived based on the reflection of light waves from the anterior and posterior corneal surfaces \\
\hline ASOCT & - Based on the emission and reflection of light (low-coherence interferometry), allowing exquisite delineation of the cornea \\
\hline Corneal tomography & $\begin{array}{l}\text { - Orbscan II: combines an advanced Placido disk system with slit-scanning technology and derives posterior surface map as well as } \\
\text { whole cornea mathematically } \\
\text { - Pentacam: uses a rotating Scheimpflug system camera with Placido disk technology to generate a three-dimensional representation } \\
\text { of the shape of the cornea } \\
\text { - Other devices: TMS-5 (Tomey Corp., Nagoya, Japan), Galilei (Ziemer, Port, Switzerland) and Sirius (CSO, Costruzione Strumenti, } \\
\text { Oftalmici, Florence, Italy) }\end{array}$ \\
\hline IVCM & $\begin{array}{l}\text { - Different layers of the cornea reflect light at different intensities, and the intensity profile Z-curve is analysed by specialised software } \\
\text { to measure the distance between any two points on the curve }\end{array}$ \\
\hline \multicolumn{2}{|c|}{ Measure corneal water concentration } \\
\hline $\begin{array}{l}\text { Terahertz scans } \\
\text { (under investigation) }\end{array}$ & - $\mathrm{THz}$ spectrum is very sensitive to water. The water content of the cornea is derived based on the reflective or absorptive THz signals) \\
\hline $\begin{array}{l}\text { Mid-infrared scans } \\
\text { (under investigation) }\end{array}$ & $\begin{array}{l}\text { - Mid-infrared radiation has very high sensitivity to the presence of the water molecule } \\
\text { - Similar to THz scans, the water concentration is estimated based on the reflected signals from the cornea }\end{array}$ \\
\hline
\end{tabular}

ASOCT $=$ anterior segment optical coherence tomography; IVCM = in vivo confocal microscopy.

or Pentacam evaluation, the accuracy depends mainly on the patient's correct gaze and alignment.

In general, the CCT measurements assessed by these instruments had highly satisfactory reproducibility, repeatability and significant linear correlation, but the readings cannot be used interchangeably. ${ }^{16}$ Conversion equations should be introduced and considered for clinical comparison of the measurements.

Besides using CCT as a surrogate marker for the severity of corneal oedema, another approach to evaluate corneal oedema is to directly quantify the water content of the cornea, which is an area in which researchers and clinicians have been starting to explore. Table 2 lists the current tools and tools under investigation for the evaluation of corneal oedema.

\section{Terahertz technology}

Terahertz ( $\mathrm{THz}$ ) waves are electromagnetic radiation in the frequency range of $0.1-10.0 \mathrm{THz}$, and $1 \mathrm{THz}$ represents $10^{12} \mathrm{~Hz}$. The most effective THz pulses are generated by a femtosecond laser, and the power used for imaging is low at $\mu \mathrm{W}$ range. ${ }^{26}$ The wavelength that the $\mathrm{THz}$ time-domain spectroscopy generates is approximately $30 \mu \mathrm{m}$, which is short enough to provide reasonable spatial resolution but long enough to prevent serious loss of signal due to scattering. The penetration depth of 1,000 um approximately. ${ }^{26,27}$ Recently, THz technology has emerged as a novel, non-invasive and non-contact tool in biomedical fields. As water has a very high dielectric constant and is highly absorptive to $\mathrm{THz}$ spectrum, $\mathrm{THz}$ waves are therefore extremely sensitive to water content in tissue, allowing its application to a variety of hydration-related diseases or conditions in different fields. ${ }^{28}$ By sensing the changes of water content in the tissue, THz technology has been used to accurately predict the skin flap failure earlier than clinical diagnosis, ${ }^{29}$ to early detect teeth that are intact but present with caries lesion, ${ }^{30}$ to identify early the features of skin cancer in skin biopsy samples, ${ }^{31}$ and to help in the diagnosis of otitis media by early detection of the presence of pus..$^{32}$

\section{The application of terahertz scans on corneas}

A cornea consists of $78 \%$ water by volume, which is the highest of any connective tissue in the body. ${ }^{1}$ The high water content of cornea, the homogeneity of corneal stromal tissue, and the relative lack of physiological variations compared to other structures in the body, allow THz imaging to be a promising method for sensing the corneal hydration level. ${ }^{33}$ Moreover, the effect of scattering from corneal collagen or extracellular matrix is less in the THz band compared to that in higherfrequency bands like infrared. ${ }^{33,34}$ As the wavelength is non-ionizing, the scanning system has been considered biologically innocuous.

The safety of $\mathrm{THz}$ scans has been demonstrated in the in vitro and animal studies. ${ }^{35-36}$ In vitro experiments demonstrated no significant effects on cell cycle kinetics and no discernible chromosomal DNA damage after exposure to $84.8 \mathrm{~mW} / \mathrm{cm}^{2}{ }^{36,37}$ Koyama et al. evaluated the in vitro cellular effects on human corneal epithelial cells following exposure to 0.12 $\mathrm{THz}$ radiation at $5 \mathrm{~mW} / \mathrm{cm}^{2}$ for 24 hours, and no significant genotoxicity or morphological changes were observed..$^{38}$ More recently, our group investigated the in vivo biological effects of continuous THz exposure with a newer version of scanning systems, in which the power density falls into the $\mu \mathrm{W}$ ranges, using a rabbit model (unpublished data). After continuous exposure of THz waves for 1, 4 and 8 hours (the actual image acquisition time for scanning is only within seconds), the corneas remained clear with no development of corneal haze or lens opacity. The corneal keratocyte activity, corneal endothelium count, and corneal thickness were evaluated using the in vivo confocal microscopy and RTvue scans, to examine whether there were negative impacts from the scanning on the corneas. After continuous exposure up to 8 hours, the quantification of stromal keratocyte reflectivity remained at the pre-scan level, indicating the THz waves did not induce activation of keratocytes. There were also no significant changes in the corneal endothelium count and corneal thickness. The corneal temperature, measured by the thermography, and the retinal rod and cone cell-mediated responses presented in the electroretinography, were not affected. Similar safety 
profiles were also shown in the histological assessments on the corneas, lens and retinas. Even after continuous scans for 8 hours, no inflammatory cells and stromal fibrotic reaction were observed in the corneas, and no necrosis, gliosis, degeneration of photoreceptors or neurons, retinal detachment, or inflammation reaction were noted in the retinal tissue. A comprehensive immunohistochemistry analysis further confirmed that there was no cellular stress response, tissue fibrogenesis, cell apoptosis or inflammatory reaction induced.

The application of $\mathrm{THz}$ sensing to the field of ophthalmology was first introduced in 2010. ${ }^{39}$ Due to a very high dielectric constant and high sensitivity to changes of water content in biological tissue, a pulsed, reflective $\mathrm{THz}$ imaging system ( $300 \mathrm{GHz}$ and $3 \mathrm{THz}$ ) can be a potential tool to measure the hydration level of the cornea. ${ }^{39}$ Bennett and his team reported that there was an approximately linear relationship between $\mathrm{THz}$ reflectivity and water concentration, using a porcine eye model..$^{28}$ The same group further conducted a feasibility study on rabbits and found a positive correlation between the $\mathrm{THz}$ reflection point signal and the corneal hydration, as well as the CCT measured by the pachymetry device. ${ }^{00,41}$ Of note, although the changes in the corneal water concentration in their experiment was only a few percent, the THz system was able to detect the difference within this interval with statistical significance. ${ }^{40}$ Lomdina et al. further reported that an $1 \%$ decrease in the content of water mass in corneas led to a clearly detectable drop of the THz reflected signal by $13 \%$, indicating good detection sensitivity. ${ }^{42}$ Similarly, our group correlated the $\mathrm{THz}$ absorptive signals of human cadaveric corneas with various extents of corneal oedema, with the CCT measured by the RTVue OCT, after adjustments for the absorption coefficient and travelling distance in the tissue. We found a strong correlation when the CCT was $<800 \mu \mathrm{m}(r=-0.77, p=0.011){ }^{43}$

The idea of using THz technology to generate hydration maps for corneas was also proposed ${ }^{40}$ although some issues have to be overcome. The sensitivity of the mapping system and the reliability of measurements of corneal water contents were first tested in human normal subjects after strengthening the imaging resolution and imaging field. ${ }^{34}$ Although the feasibility was well demonstrated, numerous practical challenges, relating to patient orientation and movement during the scanning, need to be addressed before more accurate quantitative analysis can be obtained.

One favourable characteristic of $\mathrm{THz}$ scanning technology is that it is relatively unaffected by ocular surface roughness, and the light waves can penetrate dry or hazy corneas, allowing the sensing of underlying tissue in a non-contact manner. Its high sensitivity to water content allows it to be a potential novel device to early diagnose the water gradient changes before corneal thickness has a measurable change by traditional devices like OCT or pachymetry. It also has the potential to monitor subtle corneal hydration changes, such as the progression in Fuchs' dystrophy, the tear film dynamic changes, or the changes of stromal hydration level intraoperatively during refractive surgery such as LASIK. Despite improvements in the system design and encouraging results in the animal studies as well as a pilot clinical study, substantial engineering efforts are required to refine the scanning system to eventually bring the $\mathrm{THz}$ technology closer to the medical practice.

Besides $\mathrm{THz}$, a similar concept of the use of mid-infrared laser was also proposed to monitor corneal hydration:27 a dry cornea was expected to reflect a larger amount of laser signal than a hydrated cornea. A strong correlation between the reflected signal and the water content of the cornea was observed in a porcine eye model, and the system had good sensitivity to detect $0.91 \%$ changes in the water concentration (by weight

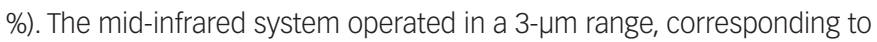
the water molecules' approximately $3300 \mathrm{~cm}^{-1}$ phonon resonance. The high photon energy implies a very shallow penetration, approximately $10 \mu \mathrm{m} .{ }^{27}$ Hence the clinical application would be mainly on the cornea surface, such as tear film dynamics.

\section{Summary}

Standard and existing evaluation tools for corneal oedema, such as ultrasound pachymetry, specular microscopy, ASOCT, Orbscan II or Pentacam, provide measurements of corneal thickness, a surrogate measure for the severity of corneal oedema. The principles of these devices are different; hence the measurements are not interchangeable, although generally a good correlation among instruments is present. The THz or mid-infrared imaging system has been emerging recently, and it provides the information on the direct water content in corneas. The preliminary data from ex vivo and in vivo corneas demonstrated a strong correlation between the reflective or absorptive signals and the CCT measured by ASOCT or pachymetry. It has the potential to detect subtle alteration in the hydration level in the cornea before the corneas start to have changes in thickness. However, research on its sensitivity and specificity is required. Generation of a hydration map, like a pachymetric map, for corneas, is also feasible, although many technical challenges have to been overcome. These light-based probing, long-wavelength sourced, and water-sensed based systems can be utilised as independent or adjuvant diagnostic and monitoring tools for the evaluation of corneal oedema. $\square$
1. RWW, Afshari N, CS B, et al. External Disease and Cornea 2016. San Fracisco, CA, USA: American Academy of Ophthalmology. 2007;14-15

2. Maurice DM. The structure and transparency of the cornea.J Physiol. 1957;136:263-86.

3. Liu YC, Wilkins M, Kim T, et al. Cataracts. Lancet. 2017;390:600-12

Farid M, Rhee MK, Akpek EK, et al. Corneal edema and opacification preferred practice pattern $\circledast$. Ophthalmolog 2019;126:P216-85.

5. Ling T, Ho A, Holden BA. Method of evaluating ultrasonic pachometers. Am J Optom Physiol Opt. 1986;63:462-6.

6. Nam SM, Im CY, Lee HK, et al. Accuracy of RTVue optical coherence tomography, Pentacam, and ultrasonic pachymetry or the measurement of central corneal thickness. . 2010;117:2096-103.

7. Osuagwu UL, Ogbuehi KC. Evaluation of the comparative effect of tetracaine on central corneal thickness measured by a contact and noncontact pachymeter J Ocul Pharmacol Ther. 2013;29:68-74

8. Yap TE, Archer TJ, Gobbe M, Reinstein DZ. Comparison of central corneal thickness between fourier-domain oct, very high-frequency digital ultrasound, and Scheimpflug imaging systems. J Refract Surg. 2016;32:110-6.

9. Reinstein DZ, Archer TJ, Gobbe M et al Repeatability of layered corneal pachymetry with the artemis very high-frequency digital ultrasound arc-scanner. J Refract Surg. 2010;26:646-59.

10. Fan R, Chan TC, Prakash G, Jhanji V. Applications of corneal topography and tomography: a review. Clin Exp Ophthalmol. 2018:46:133-46.

11. Quisling S, Sjoberg S, Zimmerman B, et al. Comparison of Pentacam and Orbscan IIz on posterior curvature topography measurements in keratoconus eyes. ophthalmology. 2006:113:1629-32.

12. Ang $\mathrm{M}$, Baskaran $\mathrm{M}$, Werkmeister RM, et al. Anterior segment optical coherence tomography. Prog Retin Eye Res. 2018;66:132-56.

13. Kaluzny BJ, Kaluzny JJ, Szkulmowska A, et al. Spectral optical coherence tomography: a novel technique for cornea imaging. coherence tomography:
Cornea. 2006;25:960-5.

14. 14. Jalbert I, Stapleton F, Papas E, et al. In vivo confoca microscopy of the human cornea. Br J Ophthalmol. 2003:87:225-36.

15. Modis L J L Langenbucher A Seitz B. Corneal thickness measurements with contact and noncontact speckness microscopic and ultrasonic pachymetry Am J Ophth 2001:132:517-21.

16. Suzuki S, Oshika T, Oki K, et al. Corneal thickness measurements: scanning-slit corneal topography and mensurements. Scanning-slt corneal topography and
noncontact specular microscopy versus ultrasonic pachymetry.

17. Zhao $\mathrm{MH}$, Zou J, Wang WQ Li J. Comparison of central cornea thickness as measured by non-contact specur microsopy thickness as measured by non-contact specular microscopy Ophthalmol. 2007:35:818-23.
18. de Sanctis U, Missolungi A, Mutani B, et al. Reproducibility and repeatability of central corneal thickness measurement in keratoconus using the rotating scheimpflug camera and ultrasound pachymetry. Am J Ophthalmol. 2007;144:712-8.

19. O'Donnell C, Maldonado-Codina C. Agreement and repeatability of central thickness measurement in normal corneas using ultrasound pachymetry and the OCULUS Pentacam. Cornea.
2005;24:920-4.

20. Barkana Y, Gerber Y, Elbaz U, et al. Central corneal thickness measurement with the Pentacam Scheimpflug system, optica low-coherence reflectometry pachymeter, and ultrasound

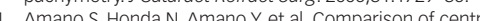

1. Amano $\mathrm{S}$, Honda $\mathrm{N}, \mathrm{Amano} \mathrm{Y}$, et al. Comparison of central corneal thickness Aeasure, ents by rotating scheimpflug topography. Ophthalmology. 2006;113:937-41.

22. Iskander NG, Anderson Penno E, Peters NT, et al. Accuracy of Orbscan pachymetry mosurements of Orbscan pachymetry measurements and DHG ultrasou LASIK enhancement las 2001;27:681-5.

3. Lackner B, Pieh S, Schmidinger G, et al. Glare and halo phenomena after laser in situ keratomileusis. J Cataract Refract phenomena after laser in situ keratomileusis. J Cataract Refract
Surg. 2003;29:444-50. 24. Salz JJ, Azen SP, Berstein J, et al. Evaluation and comparison of with utrasonic and optical machymenters ophthea thickness 
1983;14:750-4.

25. Rashid RF, Farhood QK. Measurement of central corneal thickness by ultrasonic pachymeter and oculus pentacam in patients with well-controlled glaucoma: hospital-based comparative study. Clin Ophthalmol. 2016;10:359-64.

26. Joo-Hiuk Son. Introduction to biomedical studies using terahertz waves. In: Son J-H, Terahertz biomedical science and technology. Florida, USA: CRC Press. 2014;1-7.

27. Joshi A, Bennett DB, Stafsudd OM. Monitoring corneal hydration with a mid-infrared (IR) laser. Ocul Surf. 2015;13:43-6.

28. Bennett DB, Taylor ZD, Tewari P, et al. Terahertz sensing in corneal tissues. J Biomed Opt. 2011,16:057003.

29. Bajwa N, Au J, Jarrahy R, et al. Non-invasive terahertz imaging of issue water content for flap viability assessment. Biomed Op Express. 2017;8:460-74.

30. Hall A, Girkin JM. A review of potential new diagnostic modalities for caries lesions. I Dent Res. 2004;83 Spec No C:C89-94.

31. Rahman A, Rahman AK, Rao B. Early detection of skin cancer via terahertz spectral profiling and $3 \mathrm{D}$ imaging. Biosens
Bioelectron. 2016;82:64-70.

32. Ji YB, Moon IS, Bark HS, et al. Terahertz otoscope and potential for diagnosing otitis media. Biomed opt Express. 2016;7:1201-9.

33. Sung S, Garritano J, Bajwa N, et al. Preliminary results of non-contact THz imaging of cornea. Proc SPIE Int SOC Opt Eng. 2015;9362. pii: 93620C.

34. Sung $\mathrm{S}$, Selvin $\mathrm{S}, \mathrm{Bajwa} \mathrm{N}$, et al. THz imaging system for in vivo human cornea. IEEE Trans Terahertz Sci Technol. 2018;8:27-37. 35. Taylor ZD, Singh RS, Bennett DB, et al. THz Medical Imaging: in vivo Hydration Sensing. IEEE Trans Terahertz Sci Techno. 2011,1.201-19.

36. Wilmink GJ, Rivest BD, Ibey BL, et al. Quantitative investigation of the bioeffects associated with terahertz radiation. Proc. SPIE 7562, Optical Interactions with Tissues and Cells XXI, 75620L (2 February 2010); https://doi.org/10.1117/12.844916

37. Scarfi MR, Romano M, Di Pietro R, et al. THz exposure of whole blood for the study of biological effects on human lymphocytes. J Biol Phys. 2003;29:171-6.

38. Koyama S, Narita E, Shimizu Y, et al. Twenty four-hour exposure to a $0.12 \mathrm{THz}$ electromagnetic field does not affect the genotoxicity, morphological changes, or expression of heat shock protein in HCE-T cells. Int J Environ Res Public Health. 2016;13. pii: E793.

39. Singh RS, Tewari P, Bourges JL, et al. Terahertz sensing of corneal hydration. Conf Proc IEEE Eng Med Biol SOC. 2010;2010:3021-4.

0. Bennett D, Taylor Z, Tewari P, et al. Assessment of corneal hydration sensing in the terahertz band: in vivo results at 100 GHz. J Biomed Opt. 2012;17:97008-1.

4. Taylor ZD, Garritano J, Sung S, et al. THz and mm-wave sensing of corneal tissue water content: in vivo sensing and imaging results. IEEE Trans Terahertz Sci Technol. 2015;5:184-96 2. Iomdina EN, Goltsman GN, Seliverstov SV, et al. Study of transmittance and reflectance spectra of the cornea and the sclera in the THz frequency range.J Blomed Opt. 2016,21:97002. 3. Yu-Chi Liu, Lin K, Zhang N, et al. The application of Terahertz scanning system on corneas. Presented at: Association fo Research in Vision and Ophthalmology Annual Meeting
Vancouver Canada, April 28-May 02, 2019. 